Thigpen, C. H. \& Cleckley, H. M. (1954) A case of multiple personality. Journal of Abnormal Social Psychology, 9, 135-151.

London Psychiatric Hospital
850 Highbury Avenue
PO Box 2532
London
Ontario N6A $4 \mathrm{HI}$
Canada

HAROLD MERSKeY

\section{Preconscious perceptual processing}

SIR: Fleminger (Journal, March 1992, 160, 293-303) has argued that abnormal perceptual processing is the cause of delusional misidentification. But a closer look suggests that a failure of preconscious processing underlies all perceptual experiences in which the subject makes a faulty interpretation of an external stimulus. Fleminger's argument applies equally to illusions, sensory distortions, delusional misinterpretations and delusional perception.

The traditional distinction between these experiences relies on the notion that the 'properties' or 'qualities' of an object are perceived in a different way to the 'identity' and the 'meaning' of the object. But this is mistaken. As Fleminger notes, perception is an active process of interpretation of stimuli. The ascription of meaning is an integral part of perception. Abnormalities of perceptual processing can occur in 'bottom-up' processing (incoming information) and 'top-down' processing ('expectancies' that predispose the subject to make a particular interpretation). Abnormalities of both kinds contribute to misperceptions.

Psychiatric disorders commonly influence 'topdown' processing. Illusions can arise from 'topdown' abnormalities of mood. For example, a person who is anxious may hear footsteps instead of the rustle of leaves. Sensory distortions can arise from 'top-down' alteration of the perceptual threshold. For example, a patient with hypomania may experience colours with unusual vividness. Delusional misinterpretations can arise from 'top-down' abnormalities of belief, for example, a patient with delusional jealousy may 'see' semen stains on the sheets. Similarly, delusional perception is an abnormal perceptual interpretation which arises from 'top-down' abnormalities of belief and emotion in delusional mood.

We all interpret, and misinterpret, using preconscious perceptual processing. Misinterpretation exists on a continuum encompassing normal experience and pathological symptoms. What distinguishes pathological from normal, perhaps, is not so much that a misinterpretation occurs, but that in 'normals' the misinterpretation is isolated and easily recognised and corrected. At the other extreme, schizophrenia causes such a pervasive abnormality of perceptual processing that all varieties of misinterpretation occur, and keep on occurring.

York Clinic

FranCES KLEMPERER

Guy's Hospital

London SE1 9RT

\section{Reconquest of the subjective}

SIR: In his recent article, van Praag (Journal, February 1992, 160, 266-271) defends the realm of the subjective in psychiatry against prevailing exclusively objective approaches. He strongly opposes the view that the notion of the 'subjective' has come to mean "a qualification incorrectly used as a substitute for "vague' or 'undefined"' (p. 268), i.e. has become "synonymous with non-operationable, nonmeasurable, non-quantifiable - a symbol of soft science at best" (p. 268). While we agree with Dr van Praag that subjective symptoms are important for psychiatric theory and practice, we think that his point is blurred by his imprecise and ill-defined concept of the subjective. A clearer conception of the subjective would, we believe, significantly strengthen Dr van Praag's thesis.

According to Dr van Praag, symptoms can be called 'subjective' for two reasons: (a) they are "diffuse" (p. 268) and "confined to the patient's experiential world, not expressed in objective behaviour, and 'atmospheric' rather than 'factual' in nature, that is, not manifesting themselves as delineated mental phenomena and not verbalised as such" (p. 267); (b) they are conceptualised in the mind of the interviewer/ observer by means of interpretation (cf. p.268).

It is apparent from the quote (as well as from the given examples) that the first criterion restates the view that the author is opposing in the first place, i.e., that 'subjective' has come to mean something vague, unreliable, soft, unclear, and non-clarifiable (and hence, something which has no place in science). Dr van Praag obviously sees the degree of 'delineation' of mental phenomena as a criterion for their degree of subjectivity. This can further be inferred by his introduction of the category of quasi-symptoms, i.e. symptoms which have not yet been properly operationalised.

If theoretically driven 'constructs' and inferences made by the observer about what is observed are a criterion of the subjective, then all science is subjective. Hence, Dr van Praag's second criterion of the 'subjective' is at best misleading. 
Although it is far beyond the scope of this brief commentary to present a comprehensive view of what may be called a proper account of the meaning of 'subjective', it must be mentioned that Descartes' distinction, though frequently attacked, still underlies most of our concepts and differentiations relevant to the point in question. The difference between 'signs' and 'symptoms', for example, is based on the fact that there is a large difference between my pain and anybody else's pain, as well as between pain and pain behaviour. Contrary to what some Wittgensteinian philosophers want to make us believe, we all have an intuitive grasp that our sensations and thoughts are accessible to us in a different manner than they are to somebody else. In fact, the clinician - whether practising internal medicine or psychiatry - is particularly aware of this difference. Every clinician struggles against this 'epistemological barrier' when trying to obtain a clear picture of any particular symptom.

Just as pain remains a (subjective) symptom when rated on a scale by the patient or by the observer, hallucinations and delusions remain (subjective) symptoms. Moreover, talking about pain or hallucinations does not make the pain or hallucinations intersubjectively more accessible. Their presence has merely been communicated. For example, why are we suspicious of studies claiming to have successfully 'treated' patients with auditory hallucinations by negative reinforcement using self-administered electric shocks? Obviously, because we distinguish not reporting hallucinations from not experiencing them. In short, neither rating a symptom, nor talking about a symptom, 'objectifies' it.

In our view, the acceptance and proper appreciation of the patient as experiencing subject is important to psychiatry and to medicine in general. The view that 'subjective' means 'atmospheric' or 'not delineated' does not help to achieve this goal. Nor does the relating of 'diffuse' and 'subjective' help in getting rid of the diffuseness. Only a clarified concept of the subjective as the realm of what is actually experienced by the patient, in Dr van Praag's words, will help to "expand and refine diagnostic concepts and corresponding psychometric instruments" (p. 270). Without a clear view of the subjective, "we end up with a severely coarsened psychiatry obsessed with the obvious [and] detached from the experiential realm" (p.270).

Klinikum der Universitat Heidelberg

MANFREd SPITZER

Psychiatrische Klinik

Voßstraße 4

6900 Heidelberg

Germany

New York
AUTHOR'S REPLY: My remarks on subjective psychopathological phenomena were pulled out of context by my critics. I reasoned that the prevailing psychiatric classification system based on the DSM-III caters to an overly objective approach, neglecting the realm of the subjective experiences. In that context I described subjective phenomena as those that are confined to the patient's experiential world, not expressed in observable behaviour, and 'atmospheric' rather than 'factual' in nature, that is, not manifesting themselves as delineated mental phenomena and not verbalised as such. Hence, they are largely disregarded in the present psychiatric taxonomy. I did not state that the degree of 'delineation' of mental phenomena is a criterion for their degree of subjectivity, nor did I infer such opinion in my exposition on quasi-subjective symptoms.

A second group of psychopathological phenomena I called subjective are those that are not communicated by the patient as such, but are inferences made by the observer/interviewer - "concepts" construed by incongruous observations. As an example I mentioned the meaning of a particular type of behaviour or utterance. I did not state that theoretically driven constructs are per definition subjective.

My paper did not, by any means, pretend to give a definitive description of the term 'subjective', yet my definition of what is nowadays called 'subjective' in (research) psychiatry seems to me accurate. The dissertation of Drs Spitzer and Schwartz does not provide evidence to the contrary.

Herman M. van Praag

Montefiore Medical Center

Department of Psychiatry

111 210th Street

Bronx

New York 10467

USA

\section{Guilt or morbid remorse?}

SIR: In their interesting discussion of the phenomenology of psychological guilt (Journal, June 1992, 160, 781-787), Berrios et al appear to use the word 'remorse' either as a defining component of the phenomenon or as a synonym. I would submit that these words have a different meaning; 'guilt' implying a consciousness of having committed a wrong, and 'remorse' implying an emotion of regret for the consequences of the wrongful act. I would further submit that the term 'morbid remorse' is a more accurate description of what is encountered in major depression. 